\title{
System identification and robust control of a portable proton exchange membrane fuel-cell system
}

\author{
Fu-Cheng Wang ${ }^{\mathrm{a}, *}$, Yee-Pien Yang ${ }^{\mathrm{a}}$, Chi-Wei Huang ${ }^{\mathrm{a}}$, \\ Hsin-Ping Chang ${ }^{b}$, Hsuan-Tsung Chen ${ }^{a}$ \\ a Department of Mechanical Engineering, National Taiwan University, Taipei, Taiwan \\ ${ }^{\mathrm{b}}$ Chung Shan Institute of Science and Technology (CSIST), Armaments Bureau, M.N.D, Taiwan \\ Received 21 September 2006; received in revised form 3 November 2006; accepted 17 November 2006
}

\begin{abstract}
This paper will discuss the application of system identification techniques and robust control strategies to a proton exchange membrane fuel-cell system. The fuel-cell system's dynamic behaviour is influenced by many factors, such as the reaction mechanism, pressure, flow-rate, composition and temperature change, and is inherently non-linear and time varying. From a system point of view, however, the system can be modelled as a two-input, two-output linear time-invariant system whose inputs are hydrogen and air flow rates, and whose outputs are cell voltage and current. On the other hand, the system's non-linearities and time-varying characteristics can be regarded as system uncertainties and disturbances that are treated by the designed robust controllers. This paper is comprised of three parts. First, system identification techniques were adopted to model the system's transfer functions. Second, the $H_{\infty}$ robust control strategies were applied to stabilise the system. Finally, the system's stability and performance were compromised by introducing weighting functions to the controller's design. From the experimental results, the designed $H_{\infty}$ robust controllers were deemed effective.
\end{abstract}

(C) 2006 Elsevier B.V. All rights reserved.

Keywords: Proton exchange membrane; Fuel-cell; Robust control; System identification

\section{Introduction}

As the levels of available fossil fuel decrease, alternative energy resources gain more and more attention. Among them, the fuel cell is an important candidate to replace traditional fuel because of its favourable features, such as low operational temperature, fast power response, high power density, low noise pollution, high system efficiency and environmental friendliness. Until recently, fuel cells have been applied to many systems such as bikes, motorcycles, vehicles, boats, etc. [14,15]. As a result, the control of fuel cells to increase system efficiency and stability is becoming more and more important. The traditional methodology for controlling fuel-cell systems is mainly linear control algorithms such as PI based diagonal controllers. However, these methods cannot fully satisfy the complex load change required for many applications, and may cause instability prob-

\footnotetext{
* Corresponding author. Tel.: +886 2 33662680; fax: +886 223631755 .

E-mail address: fcw@ntu.edu.tw (F.-C. Wang).
}

lems. Therefore, more elegant control strategies, such as robust control, should be applied.

Rodatz et al. [1] presented a dynamic model of air supply and developed an LQG regulator for a proton exchange membrane (PEM) fuel-cell system. From those results, performance improvements with the PI controller were noted. Moreover, the pressure trace was successfully decoupled from the mass flow trace, while a faster response time was also achieved. Pukrushpan et al. [2] developed an observer-based feedback controller to protect the fuel-cell stack from oxygen starvation during changes in current commands, while the linear quadratic technique was employed based on the linearised state-space model. Sedghisigarchi and Feliachi [3] developed an $H_{\infty}$ controller to regulate the system's output voltage under small load variations. In their simulations, the output voltage variation was kept below $5 \%$ by controlling the hydrogen flow rate. Caux et al. [4] were able to control the air supply of a proton exchange membrane fuelcell (PEMFC) system under varying current loadings. Those simulations proposed a species balance model to maintain constant pressure on the cathode (oxygen) compartment and to 
follow a desired air flow-rate. Takeuchi et al. [5] emphasized the power management of fuel-cell systems interconnected with a utility grid, while successfully introducing typical topologies of fuel-cell based distributed generation systems. Jurado and Saenz [6] developed an adaptive controller of a fuel-cell micro-turbine hybrid power plant. Considering the models' time-varying dynamics and the plants' disturbances, the adaptive controller designed at a fixed operating point was utilised to stabilise the system under different operating conditions.

The fuel-cell system demonstrated in this paper is designed and manufactured by Chung Shan Institute of Science and Technology (CSIST) and assembled by DELTA Electronics. It consists of 15 cells that are connected with pre-treated Nafion ${ }^{\circledR}$ 112 membranes under a hot press process for optimum conditions. The platinum loading is about $0.2 \mathrm{mg} \mathrm{cm}^{-2}$ at the anode and $0.4 \mathrm{mg} \mathrm{cm}^{-2}$ at the cathode. The cells are electrically connected in series. The system's inputs are hydrogen and air, while its outputs are cell voltage and current.

The fuel-cell system's dynamics is non-linear and time varying. When the current load is changed, the voltage varies from 8.5 to $12 \mathrm{~V}$ as shown in Fig. 1, using the on-board controller. Because a steady power source is important for electrical equipment, however, robust control methodologies are selected in order to provide a steady voltage or current when the operating conditions change. Robust control is well known for its capability of dealing with system uncertainties and disturbances $[8-10,12]$. Furthermore, the implementation of robust controllers is rather simple because the parameters are constant. Compared to other advanced control strategies such as adaptive or gain-scheduling control [13], in which the parameters need to be updated according to the system's responses, robust controllers are much easier to implement.

This paper is arranged in the following sections. In Section 2 , the fuel-cell system's dynamics is introduced, and further simplified as an SISO linear system by the system identification techniques, in which the un-modelled dynamics is treated as system variations. Section 3 discusses robust control strategies and their design to achieve the systems' maximum stability bounds. Section 4 introduces weighting functions and explains

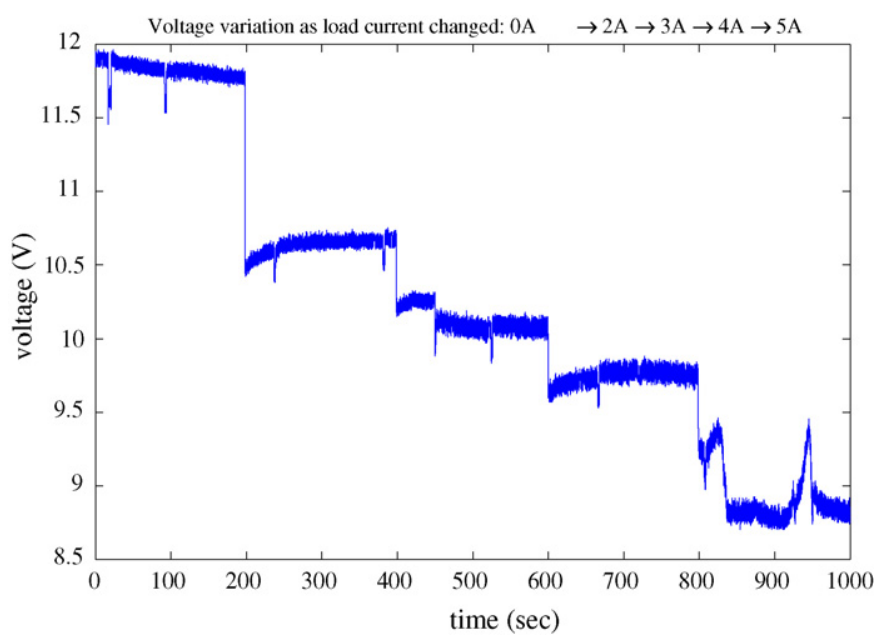

Fig. 1. The voltage variation as the load current is changed. their improvement of closed-loop systems' overall performance. Finally, Section 5 discusses some conclusions that have been drawn from this analysis.

\section{System identification of the fuel-cell system}

In this section, the fuel-cell system will be introduced and simplified as a linear model. The system transfer functions are obtained by a dynamic signal analyser, HP 35670A, and will then be used for the controller design in the following sections.

\subsection{System description}

The fuel-cell stack is designed and manufactured by CSIST and assembled by DELTA Electronics. It consists of 15 cells with the active area of $50 \mathrm{~cm}^{2}$ on each on them. The cells are connected with a pre-treated membrane - Nafion ${ }^{\circledR} 112$ - by hot press for optimum conditions. Platinum loading is about $0.2 \mathrm{~m} \mathrm{~cm}^{-2}$ at anode and $0.4 \mathrm{mg} \mathrm{cm}^{-2}$ at cathode. The cells are electrically connected in series, with rated and peak power outputs of $117 \mathrm{~W}$ at $9 \mathrm{~V}$ and $124 \mathrm{~W}$ at $7.8 \mathrm{~V}$, respectively. The maximum efficiency of the fuel cell stack is $37 \%$ (LHV) under dry $\mathrm{H}_{2}$ /air and humidification-free conditions.

The dynamics of the fuel-cell system is influenced by many factors, including the diffusion dynamic, the Nernst equation, proton concentration dynamics and cathode kinetics as illustrated in Fig. 2:

Diffusion dynamic : $R_{\mathrm{ohm}}=R_{\mathrm{ref}}+\alpha_{\mathrm{T}}\left(T-T_{\mathrm{ref}}\right)$,

Nernst equation :

$$
E=E_{\mathrm{ref}}+\frac{\mathrm{d} E^{0}}{\mathrm{~d} T}\left(T-T_{\mathrm{ref}}\right)+k \frac{R T}{2 F} \ln \left(P_{\mathrm{H}_{2}} P_{\mathrm{O}_{2}}^{1 / 2}\right),
$$

Proton concentration dynamics :

$$
u\left(-\frac{\partial c_{\mathrm{H}^{+}}}{\partial t}\right) \frac{\partial c_{\mathrm{H}^{+}}}{\partial t}+\frac{c_{\mathrm{H}^{+}}}{\tau_{\mathrm{H}^{+}}}=\frac{1+\alpha_{\mathrm{H}^{+}} j^{3}}{\tau_{\mathrm{H}^{+}}},
$$

Cathode kinetics $: \eta=b \ln \left\{\frac{P_{10}}{P_{1}} \frac{\left[H^{+}\right]_{0}}{H^{+}}\left(1+\frac{j_{\mathrm{r}}}{j_{0} A_{\mathrm{r}}}\right)\right\}$.

From this point of view, the system can be represented as the MIMO block diagram shown in Fig. 3 with the following

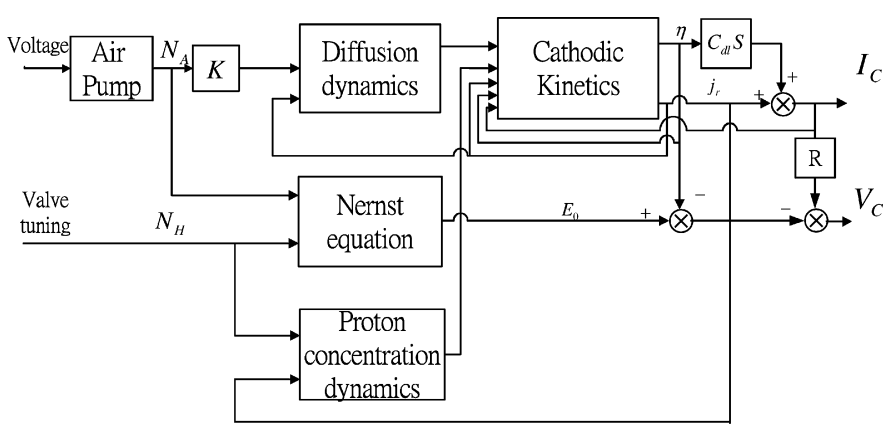

Fig. 2. The dynamics of the fuel cell system. 


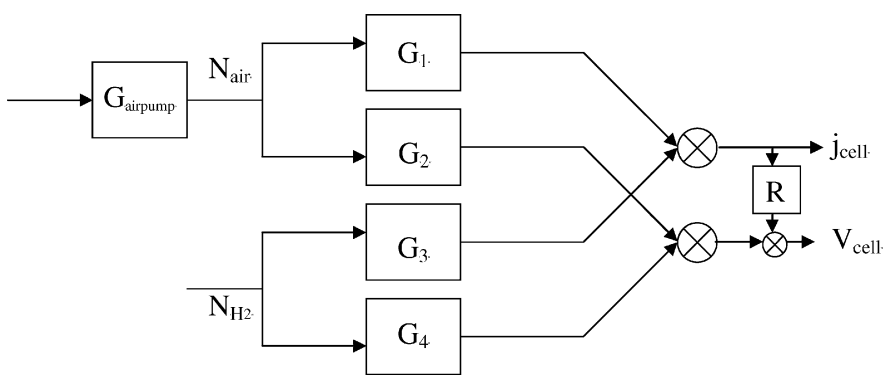

Fig. 3. The block diagrams of the fuel cell system.

relations [11]:

$j_{\text {cell }}=G_{1} N_{\text {air }}+G_{3} N_{\mathrm{H}_{2}}$,

$V_{\text {cell }}=G_{2} N_{\text {air }}+G_{4} N_{\mathrm{H}_{2}}+R j_{\text {cell }}$,

where $G_{1}-G_{4}$ represent the input-output relations of the system. Since the dynamics of the fuel-cell system is non-linear and time varying, the system of (5) and (6) is inherently non-linear and time varying as well. By fixing the hydrogen input and current output, the system is further simplified as a SISO system in the following:

$V_{\text {cell }}=G_{2} N_{\text {air }}$.

\subsection{System identification}

The non-linear system $G_{2}$ of (7) can be approximated by linear models at various operating points, given the input and output signals of the system. When considering a linear system with the sinusoidal input $r=\alpha \cos (\omega t)$, as shown in Fig. 4, the output $y$ are measured at $N$ sampled points such that the system transfer functions can be calculated by the system identification method described as follows [7]:

$I_{c}=\frac{1}{N} \sum_{t=1}^{N} y(t) \cos (\omega t)$,

$I_{s}=\frac{1}{N} \sum_{t=1}^{N} y(t) \sin (\omega t)$,

$\left|\hat{G}_{N}\left(\mathrm{e}^{\mathrm{j} \omega}\right)\right|=\frac{\sqrt{I_{c}^{2}(N)+I_{s}^{2}(N)}}{\alpha / 2}$,

$\hat{\phi}_{N}(\mathrm{j} \omega)=\arg \hat{G}_{N}\left(\mathrm{e}^{\mathrm{j} \omega}\right)=-\arctan \frac{I_{s}(N)}{I_{c}(N)}$,

where $\left|\hat{G}_{N}\left(\mathrm{e}^{\mathrm{j} \omega}\right)\right|$ and $\hat{\varphi}_{N}(\mathrm{j} \omega)$ are the gain and phase of the system at frequency $\omega$.

To model the system transfer function more efficiently, a Dynamic Signal Analyzer (DSA) HP 35670A is utilized. At first, a swept-sine analogue voltage signal, which is generated from the analyser, is sent to both of the air pump and channel-one of the

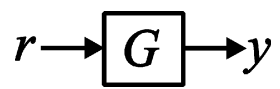

Fig. 4. A linear system.
Table 1

The operating conditions of the system

\begin{tabular}{lll}
\hline Load (A) & Input & \\
\cline { 2 - 3 } & $\mathrm{H}_{2}$ & Air \\
\hline 2 & $1.2(\mathrm{LPM}), 7 \mathrm{psi}$ & $\begin{array}{l}50 \mathrm{mHz}-100 \mathrm{~Hz} \text { sine wave } \\
\text { (level: } 2 \mathrm{~V}, \text { offset: } 4 \mathrm{~V})\end{array}$ \\
3 & $1.2(\mathrm{LPM}), 7 \mathrm{psi}$ & $\begin{array}{l}50 \mathrm{mHz}-100 \mathrm{~Hz} \text { sine wave } \\
(\text { level: } 2 \mathrm{~V}, \text { offset: } 4 \mathrm{~V})\end{array}$ \\
4 & $1.2(\mathrm{LPM}), 7 \mathrm{psi}$ & $\begin{array}{l}50 \mathrm{mHz}-100 \mathrm{~Hz} \text { sine wave } \\
(\text { level: } 2 \mathrm{~V}, \text { offset: } 5 \mathrm{~V})\end{array}$ \\
\hline
\end{tabular}

DSA to obtain the mathematical models of the system. Since the current of the input signal is in the scale of $1 \mathrm{~mA}$, while the driving current of the air-pump measures approximately $500 \mathrm{~mA}$, a power amplifier circuit is constructed to actuate the air pump. Under different operating conditions, such as those illustrated in Table 1, the output voltage of the fuel-cell system can be connected to channel-two of the DSA for system identification. Furthermore, to capture the system dynamics, the frequency range of input signals is set at between 0.05 and $100 \mathrm{~Hz}$ with 400 sampled frequencies. At each sampled frequency, the system gain and phase are calculated from (8) to (11), allowing the system transfer functions to be estimated from the frequency response diagrams. The experimental set-up of the system is illustrated in Fig. 5.

The frequency responses diagrams (Bode plots) of the fuelcell system, under different loading conditions ( $2 \mathrm{~A}, 3 \mathrm{~A}$ and 4 A), are illustrated in Fig. 6. Here, the approximate linear transfer functions of first-, second- and third-order are selected to fit the frequency responses appropriately. It is noted that the air-pump dynamics was also modelled in the transfer functions during the identification procedures. It is arranged in this way because the air-pump dynamics cannot be singled out in the control process. Lower-order models of the system can be used if the high frequency responses are insignificant for the systems.

Three experiments were executed for each operation conditions to consider the system variations: the resulting transfer functions are illustrated in Tables 2-5. Those linearised models will be used for a discussion of robust controller design in the following sections.

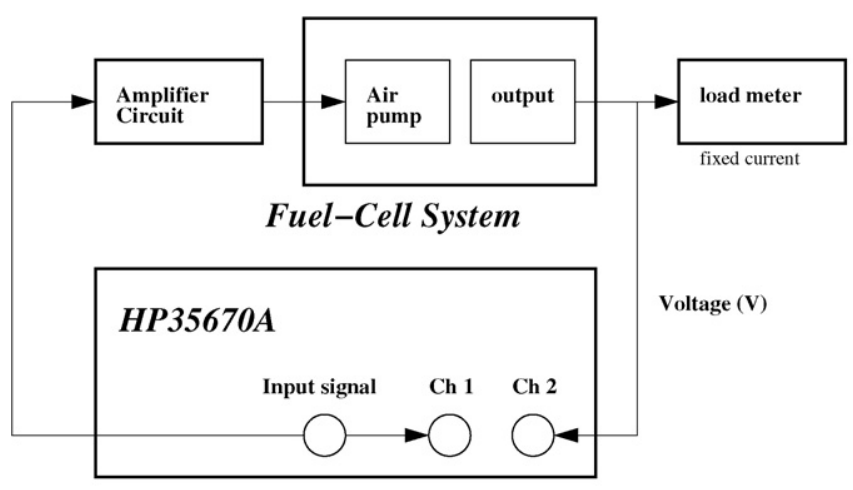

Fig. 5. The experimental setup for system identification. 
Load:2A
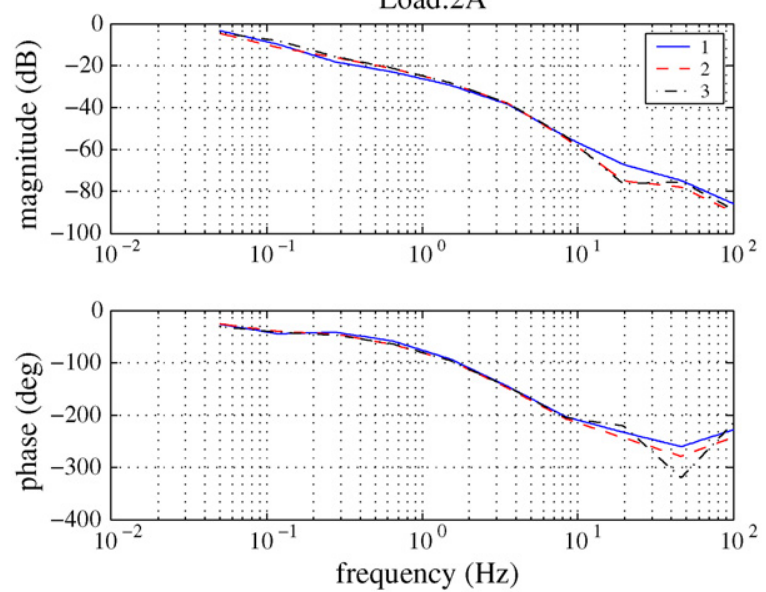

(a)
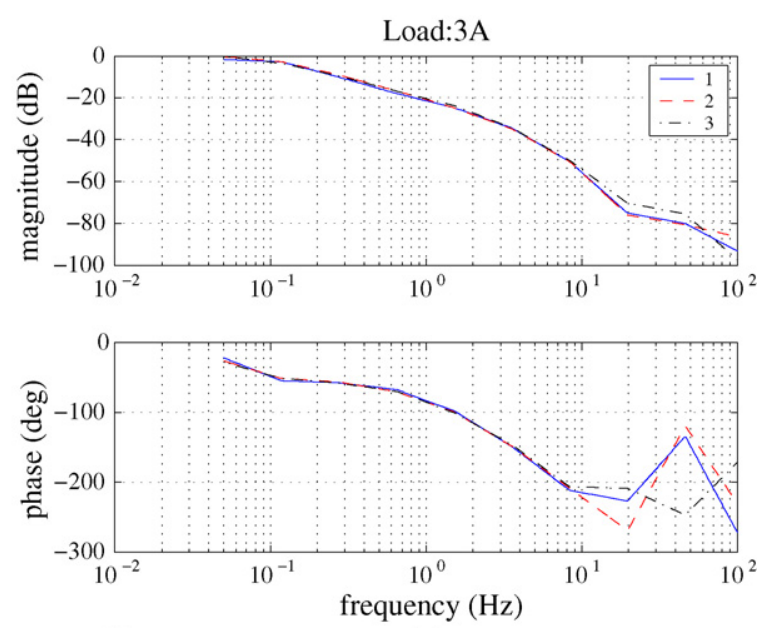

(b)

3A.

Load:4A
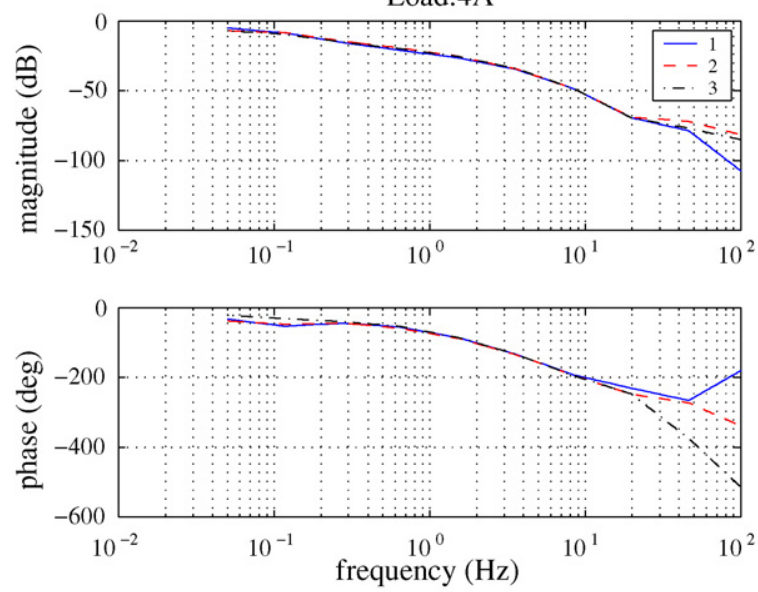

(c)

4 A.

Fig. 6. The Bode plots of the system under three different operation conditions.

Table 2

Identification of the first-order transfer functions

\begin{tabular}{lllr}
\hline No. & \multicolumn{2}{l}{ Load (A) } & \\
\cline { 2 - 4 } & 2 & 3 & \\
\hline 1 & $G_{11}=\frac{0.0010 s+0.2630}{s+0.2741}$ & $G_{21}=\frac{0.0170 s+0.6192}{s+0.6501}$ & $G_{31}=\frac{0.0014 s+0.3254}{s+0.5244}$ \\
2 & $G_{12}=\frac{0.0023 s+0.2502}{s+0.3521}$ & $G_{22}=\frac{0.0181 s+0.6427}{s+0.5913}$ & $G_{32}=\frac{0.0023 s+0.3895}{s+0.8171}$ \\
3 & $G_{13}=\frac{0.0050 s+0.3294}{s+0.4557}$ & $G_{23}=\frac{0.0129 s+0.5959}{s+0.5501}$ & $G_{33}=\frac{0.0001 s+0.3386}{s+0.7354}$ \\
\hline
\end{tabular}

Table 3

Identification of the second-order transfer functions

\begin{tabular}{lllr}
\hline No. & \multicolumn{1}{l}{ Load (A) } & & \\
\cline { 2 - 4 } & 2 & 3 & 4 \\
\hline 1 & $G_{11}=\frac{0.0008 s^{2}+0.2372 s+0.0198}{s^{2}+0.3187 s+0.0438}$ & $G_{21}=\frac{0.0086 s^{2}+0.4856 s+0.5118}{s^{2}+1.2193 s+0.6338}$ & $G_{31}=\frac{0.0040 s^{2}+0.3774 s+0.9233}{s^{2}+3.4565 s+1.4539}$ \\
2 & $G_{12}=\frac{0.0029 s^{2}+0.3073 s+0.1879}{s^{2}+1.2324 s+0.2176}$ & $G_{22}=\frac{0.0052 s^{2}+0.4289 s+2.6402}{s^{2}+4.391 s+2.5485}$ & $G_{32}=\frac{0.0043 s^{2}+0.4298 s+0.5780}{s^{2}+2.4117 s+1.1781}$ \\
3 & $G_{13}=\frac{0.0039 s^{2}+0.3160 s+0.3955}{s^{2}+1.6182 s+0.5636}$ & $G_{23}=\frac{0.0010 s^{2}+0.2181 s+9.5332}{s^{2}+16.1856 s+8.9642}$ & $G_{33}=\frac{0.0051 s^{2}+0.4499 s+0.9693}{s^{2}+3.9035 s+1.9979}$ \\
\hline
\end{tabular}


Table 4

Identification of the third-order transfer functions

No. $\quad$ Load $(\mathrm{A})$

\begin{tabular}{lllll}
\hline 2 & 3 & $4 \mathrm{~A}$ & \\
\hline 1 & $G_{11}=\frac{0.0026 s^{3}+0.2806 s^{2}+0.5943 s+0.1943}{s^{3}+2.8472 s^{2}+1.3094 s+0.2734}$ & $G_{21}=\frac{0.0049 s^{3}+0.4163 s^{2}+2.6487 s+0.4103}{s^{3}+5.3781 s^{2}+5.1796 s+2.0519}$ & $G_{31}=\frac{0.0023 s^{3}+0.3263 s^{2}+5.4754 s+11.1666}{s^{3}+15.2201 s^{2}+43.5656 s+17.4807}$ \\
2 & $G_{12}=\frac{0.0036 s^{3}+0.3129 s^{2}+0.8772 s+0.9834}{s^{3}+3.1372 s^{2}+5.2340 s+1.2132}$ & $G_{22}=\frac{0.0015 s^{3}+0.2330 s^{2}+6.4609 s+2.5733}{s^{3}+10.9154 s^{2}+9.9708 s+2.6322}$ & $G_{32}=\frac{0.0031 s^{3}+0.4162 s^{2}+2.9779 s+2.7421}{s^{3}+8.1131 s^{2}+13.3834 s+5.6468}$ \\
3 & $G_{13}=\frac{0.0017 s^{3}+0.2556 s^{2}+2.9615 s+0.4103}{s^{3}+9.7338 s^{2}+5.3668 s+0.6996}$ & $G_{23}=\frac{0.0002 s^{3}+0.0492 s^{2}+12.7968 s+0.2916}{s^{3}+21.9285 s^{2}+12.1025 s+0.1491}$ & $G_{33}=\frac{0.0003 s^{3}+0.1219 s^{2}+10.6176 s+33.8400}{s^{3}+22.8709 s^{2}+126.5667 s+70.0607}$ \\
\hline
\end{tabular}

Table 5

The gap metric and stability margin of the first-order systems (with minimization of the maximum gap and the corresponding stability bound in boxes)

\begin{tabular}{|c|c|c|c|c|c|c|c|c|c|c|}
\hline & $G_{11}$ & $G_{12}$ & $G_{13}$ & $G_{21}$ & $G_{22}$ & $G_{23}$ & $G_{31}$ & $G_{32}$ & $G_{33}$ & $b(G, K)$ \\
\hline$G_{11}$ & 0.0000 & 0.1465 & 0.1391 & 0.2829 & 0.3076 & 0.2837 & 0.2081 & 0.3150 & 0.3274 & 0.9280 \\
\hline$G_{12}$ & 0.1465 & 0.0000 & 0.0805 & 0.2887 & 0.3202 & 0.2992 & 0.0789 & 0.1727 & 0.1862 & 0.9530 \\
\hline$G_{13}$ & 0.1391 & 0.0805 & 0.0000 & 0.2115 & 0.2451 & 0.2257 & 0.0707 & 0.1808 & 0.1932 & 0.9522 \\
\hline$G_{21}$ & 0.2829 & 0.2887 & 0.2115 & 0.0000 & 0.0664 & 0.0643 & 0.2288 & 0.3117 & 0.3238 & 0.9316 \\
\hline$G_{22}$ & 0.3076 & 0.3202 & 0.2451 & 0.0664 & 0.0000 & 0.0295 & 0.2738 & 0.3730 & 0.3859 & 0.9193 \\
\hline$G_{23}$ & 0.2837 & 0.2992 & 0.2257 & 0.0643 & 0.0295 & 0.0000 & 0.2671 & 0.3716 & 0.3846 & 0.9186 \\
\hline$G_{31}$ & 0.2081 & 0.0789 & 0.0707 & 0.2288 & 0.2738 & 0.2671 & 0.0000 & 0.1110 & 0.1241 & 0.9619 \\
\hline$G_{33}$ & 0.3274 & 0.1862 & 0.1932 & 0.3238 & 0.3859 & 0.3846 & 0.1241 & 0.0314 & 0.0000 & 0.9768 \\
\hline Max. & 0.3274 & 0.3202 & 0.2451 & 0.3238 & 0.3859 & 0.3846 & 0.2738 & 0.3730 & 0.3859 & \\
\hline
\end{tabular}

Table 6

The gap metric and stability margin of the second-order systems (with minimization of the maximum gap and the corresponding stability bound in boxes)

\begin{tabular}{|c|c|c|c|c|c|c|c|c|c|c|}
\hline & $G_{11}$ & $G_{12}$ & $G_{13}$ & $G_{21}$ & $G_{22}$ & $G_{23}$ & $G_{31}$ & $G_{32}$ & $G_{33}$ & $b(G, K)$ \\
\hline$G_{11}$ & 0.0000 & 0.2844 & 0.1869 & 0.3292 & 0.3696 & 0.3826 & 0.1471 & 0.2407 & 0.2452 & 0.9406 \\
\hline$G_{12}$ & 0.2844 & 0.0000 & 0.1082 & 0.3248 & 0.3323 & 0.3096 & 0.1463 & 0.2537 & 0.2582 & 0.9459 \\
\hline$G_{13}$ & 0.1869 & 0.1082 & 0.0000 & 0.2465 & 0.2488 & 0.2218 & 0.0469 & 0.1555 & 0.1600 & 0.9527 \\
\hline$G_{21}$ & 0.3292 & 0.3248 & 0.2465 & 0.0000 & 0.1240 & 0.1373 & 0.2649 & 0.2792 & 0.3020 & 0.9283 \\
\hline$G_{22}$ & 0.3696 & 0.3323 & 0.2488 & 0.1240 & 0.0000 & 0.0372 & 0.2731 & 0.3401 & 0.3445 & 0.9126 \\
\hline$G_{23}$ & 0.3826 & 0.3096 & 0.2218 & 0.1373 & 0.0372 & 0.0000 & 0.2569 & 0.3524 & 0.3568 & 0.9135 \\
\hline$G_{31}$ & 0.1471 & 0.1463 & 0.0469 & 0.2649 & 0.2731 & 0.2569 & 0.0000 & 0.1095 & 0.1141 & 0.9622 \\
\hline$G_{33}$ & 0.2452 & 0.2582 & 0.1600 & 0.3020 & 0.3445 & 0.3568 & 0.1141 & 0.0320 & 0.0000 & 0.9776 \\
\hline Max. & 0.3826 & 0.3323 & 0.2488 & 0.3292 & 0.3696 & 0.3826 & 0.2731 & 0.3524 & 0.3568 & \\
\hline
\end{tabular}

Table 7

The gap metric and stability margin of the third-order systems (with minimization of the maximum gap and the corresponding stability bound in boxes)

\begin{tabular}{|c|c|c|c|c|c|c|c|c|c|c|}
\hline & $G_{11}$ & $G_{12}$ & $G_{13}$ & $G_{21}$ & $G_{22}$ & $G_{23}$ & $G_{31}$ & $G_{32}$ & $G_{33}$ & $b(G, K)$ \\
\hline$G_{11}$ & 0.0000 & 0.0907 & 0.1062 & 0.3274 & 0.3266 & 0.4627 & 0.1281 & 0.2219 & 0.2164 & 0.9412 \\
\hline$G_{12}$ & 0.0907 & 0.0000 & 0.1509 & 0.3238 & 0.3351 & 0.4059 & 0.1134 & 0.2277 & 0.2299 & 0.9488 \\
\hline$G_{13}$ & 0.1062 & 0.1509 & 0.0000 & 0.2453 & 0.2528 & 0.5382 & 0.0503 & 0.1407 & 0.1436 & 0.9549 \\
\hline$G_{22}$ & 0.3266 & 0.3351 & 0.2528 & 0.1074 & 0.0000 & 0.3189 & 0.2796 & 0.3289 & 0.3403 & 0.9131 \\
\hline$G_{23}$ & 0.4627 & 0.4059 & 0.5382 & 0.4183 & 0.3189 & 0.0000 & 0.5054 & 0.6021 & 0.6039 & 0.9052 \\
\hline$G_{31}$ & 0.1281 & 0.1134 & 0.0503 & 0.2624 & 0.2796 & 0.5054 & 0.0000 & 0.1168 & 0.1188 & 0.9618 \\
\hline Max. & 0.4627 & 0.4059 & 0.5382 & 0.4183 & 0.3403 & 0.6039 & 0.5054 & 0.6021 & 0.6039 & \\
\hline
\end{tabular}




\section{Robust stability design}

In this section, robust control strategies will be introduced. From the analyses of gap metrics and coprime factorisation, robust controllers are designed to provide the maximum stability bound for the fuel-cell system. The resulting controllers are then verified from both simulations and experiments.

\subsection{Principles of robust control}

Robust controllers can be designed for the identified linear models by using robust control strategies, such as Small Gain Theorem, perturbations of coprime factors, stability margin and gap metric [8-10]. A system is said to be well-posed if all closedloop transfer matrices are well-defined and proper. Furthermore, a closed-loop system is said to be internally stable if all the system states are asymptotically stable, i.e., the states go to zero from all initial state conditions when the input signals are zero.

Theorem 1 (Small Gain Theorem [8]). Suppose $M \in R H_{\infty}$ and let $\gamma>0$. Then the interconnected system shown in Fig. 7 is well-posed and internally stable for all $\Delta(s) \in R H_{\infty}$ with

(a) $\|\Delta\|_{\infty} \leq 1 / \gamma$ if and only if $\|M(s)\|_{\infty}<\gamma$;

(b) $\|\Delta\|_{\infty}<1 / \gamma$ if and only if $\|M(s)\|_{\infty}<\gamma$,

where $\|G\|_{\infty}$ is the $\infty$ norm of system $G$.

Suppose that a nominal plant $G_{0}$ can be expressed as $G_{0}=M N^{-1}$, where (1) $M, N \in R H_{\infty}$ and (2) $M M^{*}+N N^{*}=I, \forall \omega$. This is called a normalised left coprime factorisation of $G_{0}$. Now, let us suppose that a perturbed system $G_{\Delta}$ with the block diagram of Fig. 8 is expressed as:

$G_{\Delta}=\left(M+\Delta_{M}\right)^{-1}\left(N+\Delta_{N}\right)$,

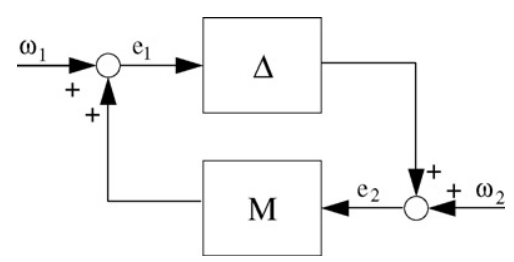

Fig. 7. Small Gain Theorem.

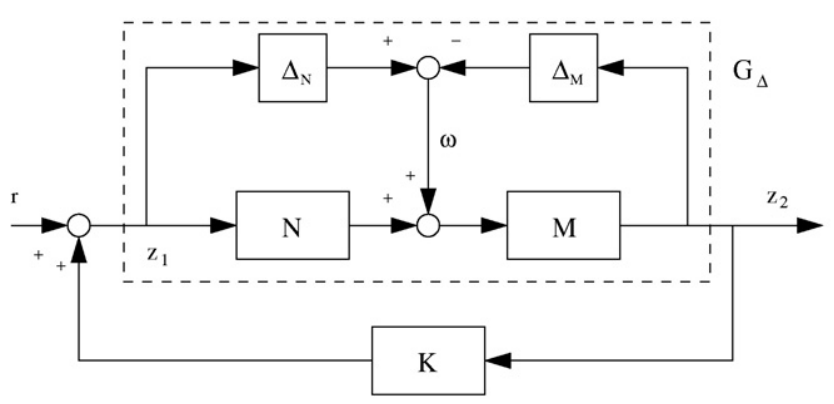

Fig. 8. The block diagram of $G_{\Delta}=\left(M+\Delta_{M}\right)^{-1}\left(N+\Delta_{N}\right)$ with feedback controller $K$. with $\left\|\left[\Delta_{M}, \Delta_{N}\right]\right\|_{\infty}<\varepsilon, \Delta_{M}, \Delta_{N} \in R H_{\infty}$, the system transfer functions can be simplified as follows:

$$
\begin{aligned}
{\left[\begin{array}{l}
z_{1} \\
z_{2}
\end{array}\right] } & =\left[\begin{array}{c}
K \\
I
\end{array}\right](I-G K)^{-1} M^{-1} \omega \\
& =\left[\begin{array}{c}
K \\
I
\end{array}\right](I-G K)^{-1}[I G] \omega .
\end{aligned}
$$

Hence, from Theorem 1, the closed-loop system remains internally stable for all $\left\|\left[\Delta_{M}, \Delta_{N}\right]\right\|_{\infty}<\varepsilon$ if and only if

$$
\begin{aligned}
& \left\|\left[\begin{array}{c}
K \\
I
\end{array}\right](I-G K)^{-1} M^{-1}\right\|_{\infty} \leq \frac{1}{\varepsilon}, \\
& \quad \text { or equivalently }\left\|\left[\begin{array}{c}
K \\
I
\end{array}\right](I-G K)^{-1}[I G]\right\|_{\infty} \leq \frac{1}{\varepsilon} .
\end{aligned}
$$

Defined the stability margin $b(G, K)$ of the closed-loop system as follows:

$b(G, K) \equiv\left\|\left[\begin{array}{l}K \\ I\end{array}\right](I-G K)^{-1}[I G]\right\|_{\infty}^{-1}$,

from Theorem 1 the closed-loop system is internally stable for all $\left\|\left[\Delta_{M}, \Delta_{N}\right]\right\|_{\infty}<\varepsilon$ if and only if $b(G, K) \geq \varepsilon$.

It is further noted that the coprime factorisation of a system is not unique. That is, there is more than one expression for $G_{0}$ and $G_{\Delta}$. Therefore, the gap between two systems $G_{0}$ and $G_{\Delta}$ can be defined as:

Definition 2 (Gap metric [8]). The smallest value of $\|\left[\Delta_{M}\right.$, $\left.\Delta_{N}\right] \|_{\infty}$ which perturbs $G_{0}$ into $G_{\Delta}$, is called the gap between $G_{0}$ and $G_{\Delta}$, and is denoted as $\delta\left(G_{0}, G_{\Delta}\right)$.

Thus, $b\left(G_{0}, K\right)$ gives the radius (in terms of the distance in the gap metric) of the largest ball of plants stabilised by controller $K$. Therefore, the design goal is to derive a suitable controller $K$ from a nominal plant $G_{0}$, such that all perturbed plants $G_{i}$ located inside the gap $\delta\left(G_{0}, G_{i}\right)<\varepsilon$ will satisfy $b\left(G_{0}, K\right) \geq \varepsilon$ and the closed-loop system remains internally stable.

\subsection{Robust controller design}

To perform robust controller design, several nominal plants are selected as follows:

First-order system, $G_{0 \_1}(s)=\frac{0.0050 s+0.3294}{s+0.4557}$,

Second-order system :

$$
G_{0 \_2}(s)=\frac{0.0039 s^{2}+0.03160 s+0.3955}{s^{2}+1.6182 s+0.5636},
$$

Third-order system :

$$
G_{0 \_3}(s)=\frac{0.0015 s^{3}+0.2330 s^{2}+6.4609 s+2.5733}{s^{3}+10.9154 s^{2}+9.9708 s+2.6322} .
$$




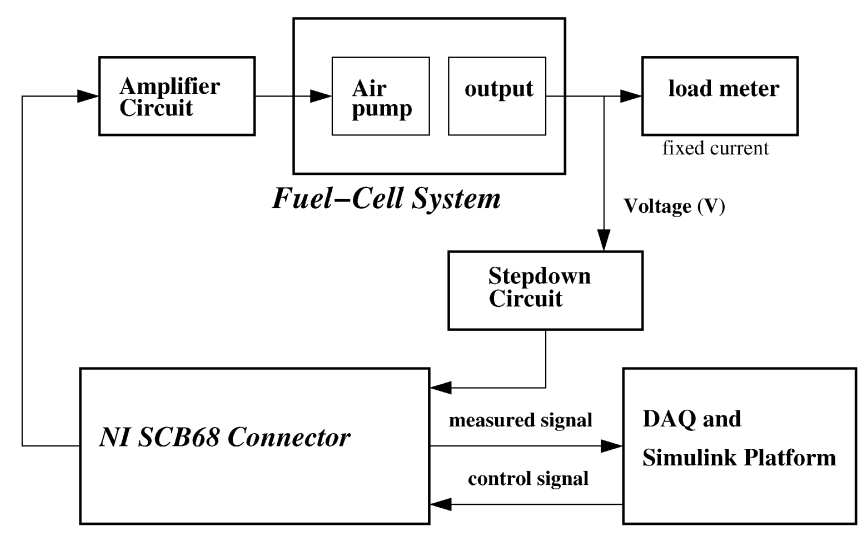

Fig. 9. The experimental setup of the PEMFC control system.

The choices of the nominal plants $G_{0}(s)$ are based on the calculation of system gaps between the nominal plants and the perturbed plants such that the maximum gap is minimized as:

$\min _{G_{0}} \max \delta\left(G_{0}, G_{i}\right)$.

For instance, for the first-order system, $G_{13}$ is chosen as the nominal plant because the maximum gap between $G_{13}$ and other plants is 0.2451 - the minimum for all systems (shown in Table 5).

By applying robust control to the nominal plants, the resulting $H_{\infty}$ controllers are:

First-order system, $K_{1}(s)=-0.3263$,

Second-order system : $K_{2}(s)=\frac{-0.3234 s-0.3776}{s+1.2079}$,
Third-order system : $K_{3}(s)=\frac{-0.4483 s^{2}-4.6502 s-1.5751}{s^{2}+11.4022 s+4.2596}$.

The stability bounds of the controllers are $b(G, K)=0.9522$, 0.9527 and 0.9131 , respectively (Tables $5-7$ ). It must be noted that the maximum gaps between the nominal plants and the perturbed plants are always less than the stability bound, such that internal stability can be achieved for all system variations by using the designed controllers.

\subsection{Experimental results}

The designed controllers were implemented to verify the effects. For the experiments, a closed-loop control system of the PEM fuel-cell system was developed by using the National Instrument's (NI) Data Acquisition (DAQ) card and Simulink. This was accomplished by transferring the control signal through the output port of the DAQ card and by measuring the voltage of the PEMFC system to the input port of the DAQ card. A step-down circuit was constructed to divide the measured fuel-cell voltage by 2 to protect the DAQ card since the allowable voltage range of the DAQ card is from -10 to $+10 \mathrm{~V}$, while the maximum voltage of the PEM fuel-cell system is about $14 \mathrm{~V}$. The experimental set-up of the PEM fuel-cell control system is illustrated in Fig. 9. The output responses of the closed-loop systems are shown in Fig. 10, where the controllers (19)-(21) were implemented to control the fuel-cell system under different loading conditions when the reference voltage was set at $9.5 \mathrm{~V}$ First of all, it is noted that the closed-loop systems remain stable under
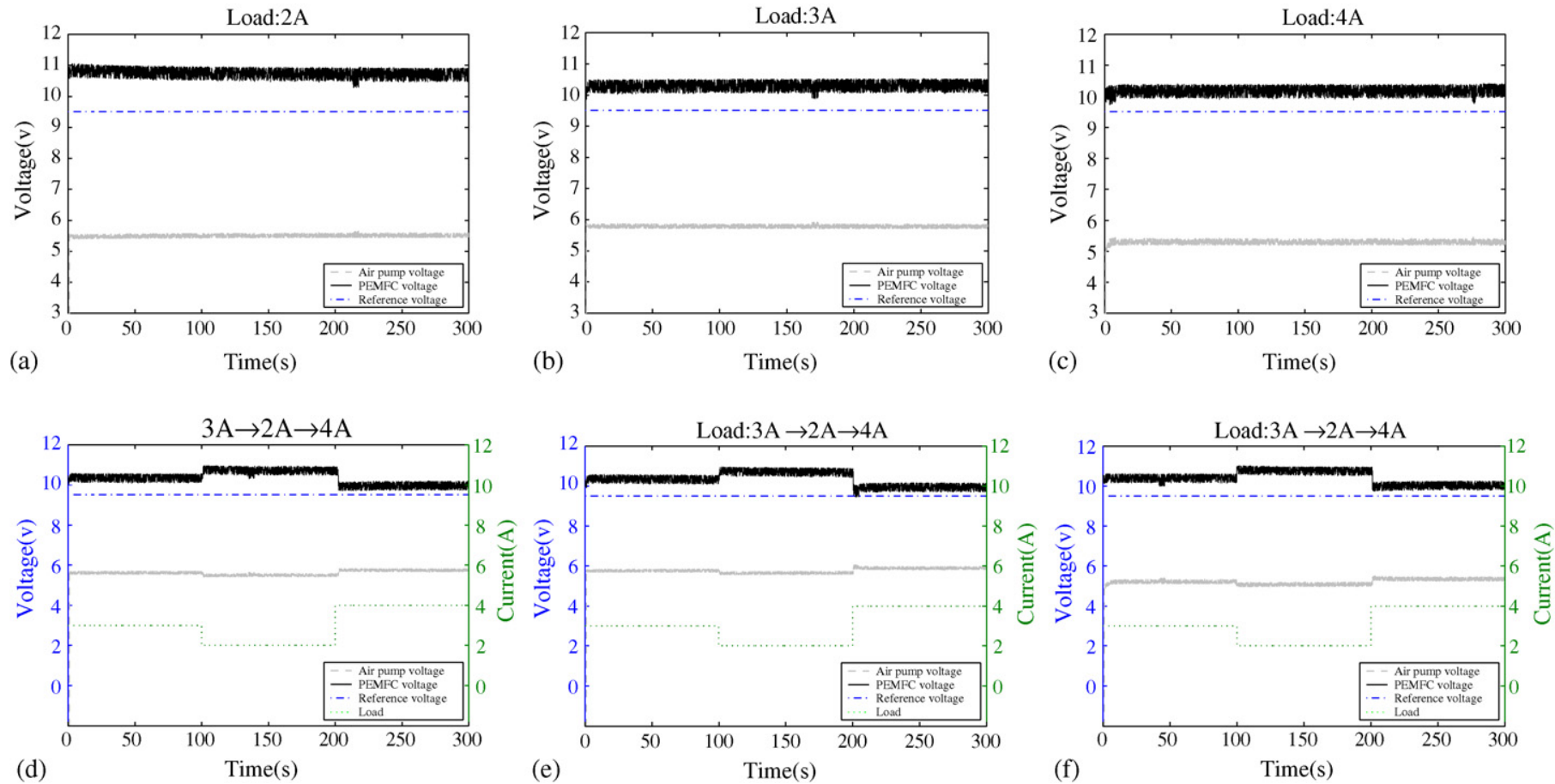

Fig. 10. The output voltage responses of (a) $K_{1}(s)$ for $2 \mathrm{~A}$ loads, (b) $K_{2}(s)$ for $3 \mathrm{~A}$ loads, (c) $K_{3}(s)$ for 4 A loads, and (d) $K_{1}(s)$, (e) $K_{2}(s)$, (f) $K_{3}(s)$ for current setting $3 \mathrm{~A} \rightarrow 2 \mathrm{~A} \rightarrow 4 \mathrm{~A}$. 


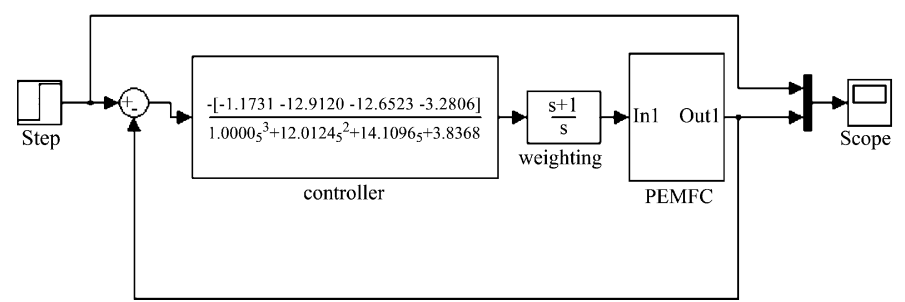

Fig. 11. The robust controller design with weighting functions.

system variations. Secondly, the controlled voltage of the airpump was 5-6 V. Finally, the output voltage was approximately $10-11 \mathrm{~V}$, while the reference (or desired) voltage was set at $9.5 \mathrm{~V}$.

From the experimental results, it is shown that the closedloop systems are stabilised by the designed robust controllers. However, it is also noted that the system performance is not satisfactory because the controllers were designed to achieve the maximum stability bound, such that system performance was relatively ignored. For example, in the experiments the reference voltage was set at $9.5 \mathrm{~V}$, but the output voltage varied from 10 to $11 \mathrm{~V}$. Therefore, it is necessary to consider the overall performance criteria by adding weighting functions into the controller design. These issues are discussed in the following section.

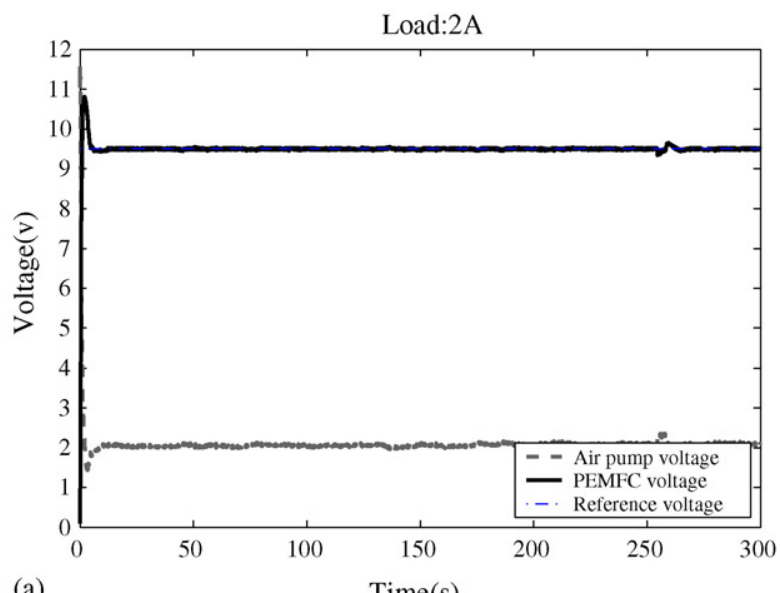

(a)

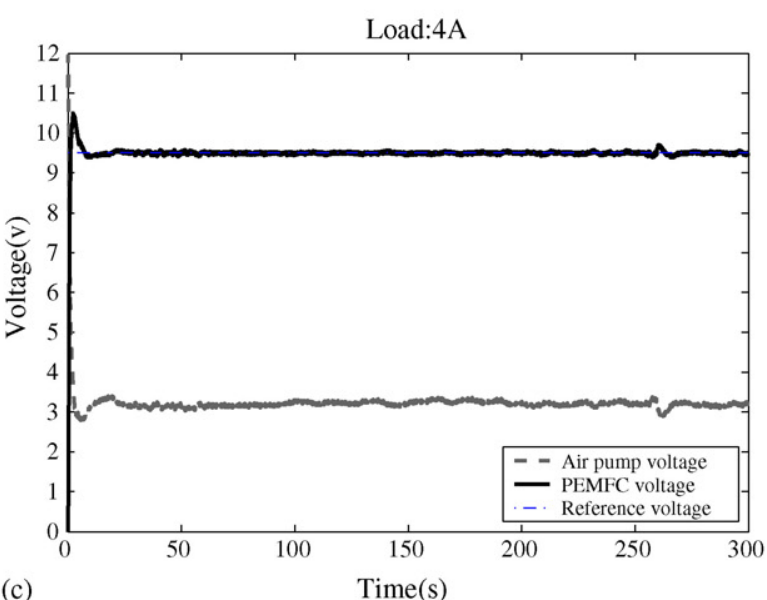

\section{Robust performance design}

The controller design discussed in Section 3 focused on the stability of the closed-loop systems: the design criterion was to discover the maximum stability bound to cope with system uncertainties. However, the system performance was relatively ignored, as shown in the system responses of Fig. 10. Therefore, in this section, weighting functions will be introduced to improve the system performance. It will be shown from the results that the system performance is significantly improved by sacrificing a little stability bound.

\subsection{Weighting function selection}

There were steady-state errors between the reference and the system outputs, as illustrated in Fig. 10. To track the reference command, an integral was added to the weighting functions. First, a weighting function $W(s)=(s+1) / s$ was added to the controller design, as illustrated in Fig. 11. For the third-order system with the nominal plant $G_{03}(s)$ of (18), the robust controller was found as:

$K_{4}(s)=\frac{1.1731 s^{3}+12.9120 s^{2}+12.6523 s+3.2806}{s^{3}+12.0124 s^{2}+14.1096 s+3.8368}$,

with a maximum stability bound $b\left(G_{0 \_3}, K_{4}\right)=0.6499$.
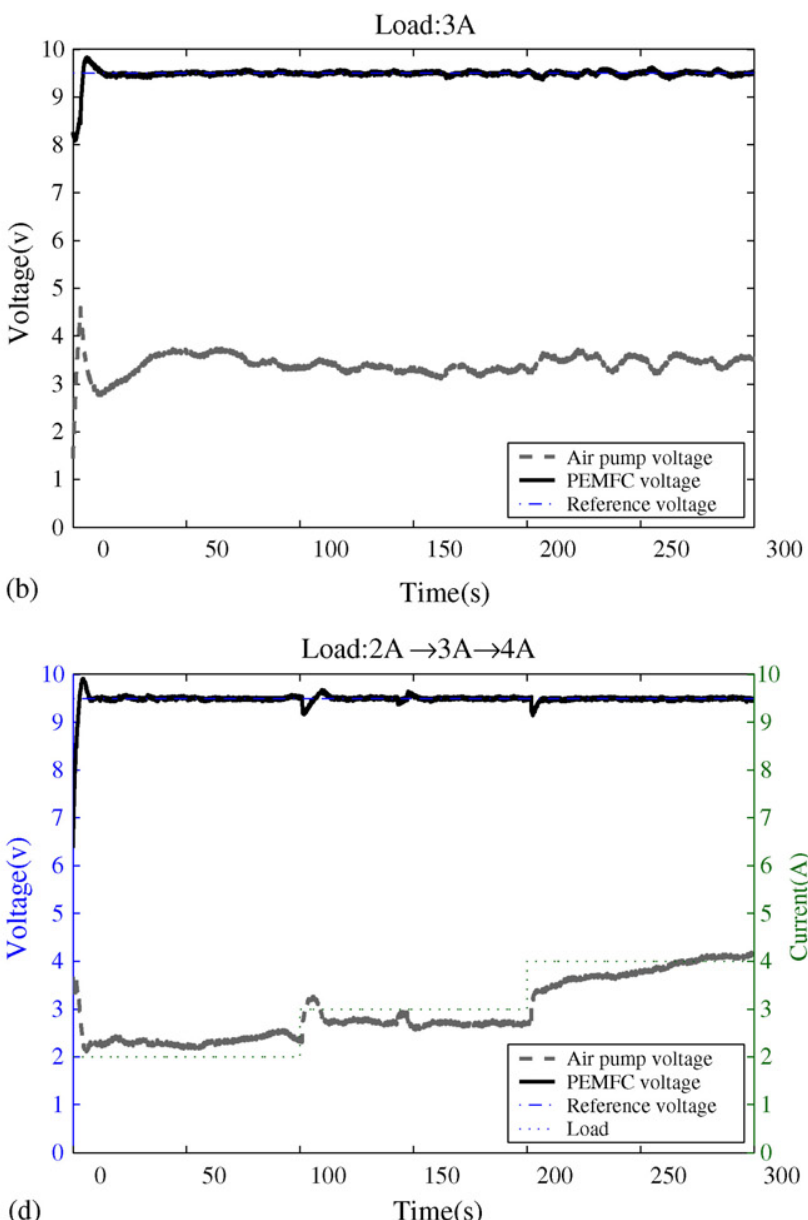

(d)

Time $(\mathrm{s})$

Fig. 12. The output voltage responses of the fuel-cell system with $K_{4}(s)$ under loadings: (a) $2 \mathrm{~A}$, (b) $3 \mathrm{~A}$, (c) $4 \mathrm{~A}$ and (d) $2 \mathrm{~A} \rightarrow 3 \mathrm{~A} \rightarrow 4 \mathrm{~A}$. 


\subsection{Experimental results}

The experimental results using the designed robust controller $K_{4}(s)$ are shown in Fig. 12, where the reference voltage is set at $9.5 \mathrm{~V}$ while the loadings are set at $2 \mathrm{~A}, 3 \mathrm{~A}, 4 \mathrm{~A}$ and $2 \mathrm{~A} \rightarrow 3 \mathrm{~A} \rightarrow 4 \mathrm{~A}$, respectively. It is shown that the closed-loop system is internally stable for system perturbations, and that the steady-state error is zero because of the integral factor of the weighting function. It is noted the use of the weighting function can be regarded as a compromise between the stability and performance. As the system performance is improved using the controller $K_{4}(s)$, the relative stability bound of the closedloop systems is decreased from $b\left(G_{0 \_}, K_{3}\right)=0.9131$ to $b\left(G_{0_{-} 3}\right.$, $\left.K_{4}\right)=0.6499$. That is, the system robustness is reduced.

\section{Conclusion}

This paper has proposed system identification techniques and robust control methods for a PEM fuel-cell system. From system identification, the fuel-cell system was modelled as an MIMO system, which was further simplified to an SISO system by fixing the hydrogen input and the current output. By selecting the nominal plants, the system variations were regarded as system uncertainties and disturbances that were treated by the proposed robust controllers. At first, a robust controller was designed to achieve the closed-loop systems' maximum stability margins. Then, weighting functions were introduced to improve the system's overall performance. The experiments have shown that the designed robust controllers can achieve good system performance and stability. Although this paper has been limited to the SISO robust controller design only, it is important to note that the principles of developing an MIMO robust controller will be similar and could be applied widely.

\section{References}

[1] P. Rodatz, G. Paganelli, L. Guzzella, Proceedings of the American Control Conference, Adams Mark Hotel, Denver, CO, June 4-6, 2003, pp. 2043-2048.

[2] J.T. Pukrushpan, H. Peng, A.G. Stefanopoulou, ASME International Mechanical Engineering Congress and Exposition, New Orleans, Louisiana, November 17-22, 2002.

[3] K. Sedghisigarchi, A. Feliachi, Proceedings of the 35th Southeastern Symposium on System Theory 16-18, 2003, pp. 464-467.

[4] S. Caux, J. Lachaize, M. Fadel, P. Schott, L. Nicod, 2005 IEEE Vehicle Power and Propulsion (VPP) Conference, Illinois Institute of Technology, Chicago, Illinois, USA, September 7-9, 2005, pp. 597-602.

[5] A. Takeuchi, N. Yamashita, T. Yachi, The 27th Annual Conference of the IEEE Industrial Electronics Society 2, Denver, CO, USA, 2001, pp. 1297-1302.

[6] F. Jurado, J.R. Saenz, IEEE Trans. Energy Convers. 18 (2) (2003) 342-347.

[7] L. Ljung, System Identification Theory for the User, Prentice-Hall, 1987.

[8] K. Zhou, J.C. Doyle, K. Glover, Robust and Optimal Control, Prentice-Hall, 1996.

[9] T.T. Georgiou, M.C. Smith, IEEE Trans. Autom. Control 35 (1990) 673-686.

[10] G. Vinnicombe, IEEE Trans. Autom. Control 38 (1993) 1371-1383.

[11] J.M. Maciejowski, Multivariable Feedback Design, Addison Wesley, 1990.

[12] F.C. Wang, Y.P. Yang, et al., Proceedings of FUELCELL2006, Irvine, CA, USA, FUELCELL2006-97118, 2006.

[13] Y.P. Yang, F.C. Wang, et al., Proceedings of FUELCELL2006, Irvine, CA, USA, FUELCELL2006-97119, 2006.

[14] D.M. Chen, H. Peng, ASME J. Dyn. Syst. Meas. Control 127 (3) (2005) 424-432.

[15] J.T. Pukrushpan, H. Peng, ASME J. Dyn. Syst. Meas. Control 126 (1) (2004) 14-25. 